\title{
Detection of multiple fluorescent labels using superconducting tunnel junction detectors
}

\author{
G. W. Fraser ${ }^{a)}$ \\ Space Research Centre, Department of Physics and Astronomy, University of Leicester, Leicester LE1 7RH, \\ United Kingdom \\ J. S. Heslop-Harrison and T. Schwarzacher \\ Department of Biology, University of Leicester, Leicester LE1 7RH, United Kingdom \\ A. D. Holland \\ Space Research Centre, Department of Physics and Astronomy, University of Leicester, Leicester LE1 7RH, \\ United Kingdom \\ P. Verhoeve and A. Peacock \\ Science Payloads Technology Division SCI-ST, Research and Scientific Support Department, Postbus 299, \\ ESA/ESTEC, 2200 AG Noordwjik, The Netherlands
}

(Received 27 January 2003; accepted 4 June 2003)

\begin{abstract}
We show that cryogenically cooled, photon counting superconducting tunnel junctions (STJs) can be used to simultaneously record the optical spectra from multiple biological fluorochromes. Measurements with a single-pixel tantalum STJ with a wavelength resolving power $R=\lambda / \Delta \lambda$ of about 10 confirm the expected sensitivity advantage with respect to the photomultiplier-based detectors commonly used to record signals from microarrays and other fluorescent biological systems. (C) 2003 American Institute of Physics. [DOI: 10.1063/1.1599059]
\end{abstract}

\section{INTRODUCTION}

The simultaneous measurement of the optical emission from collocated fluorochromes is a common problem in biology. Imaging cell components demands the temporal registration of multiple fluorescent markers such as variants of green fluorescent protein (GFP). In fluorescent resonant energy transfer (FRET), changes in emission spectra indicate the physical separation between donor and acceptor dye molecules. Quantifying the hybridization of labeled nucleic acids (probes) to immobilized target molecules in microarray format or in cells in situ ideally requires the efficient, artifactfree measurement of several fluorescent spectra simultaneously. ${ }^{1}$ Cryogenically cooled superconducting tunnel junction (STJ) detectors ${ }^{2-6}$ developed for astronomy measure the energies of individual optical photons with very low levels of internal background. The operation of a STJ is indicated schematically in Fig. 1(a). In this article, we show that STJs can be used for the sensitive, quantitative registration of multiple biological fluorochromes. Using a single near-UV excitation filter, and standard dichroic and longpass filters, the emission spectra of common fluorochromes could be easily distinguished - from each other and from broadband substrate fluorescence. STJ detectors have the potential to revolutionize quantitative multifluorochrome imaging in biology.

Photomultiplier tubes (PMTs) and image intensifiers are the photon-counting detectors most commonly used to image weakly fluorescent biological specimens. These detectors

\footnotetext{
a) Author to whom correspondence should be addressed; electronic mail: gwf@star.le.ac.uk
}

provide information on photon color (energy or wavelength) only when used with narrow band output filters or dispersive gratings. Their efficiency is limited by the quantum yield $Q$ electrons/photon of the PMT photocathode. For a photocathode such as $\mathrm{S} 20(\mathrm{CsKNaSb}){ }^{7} Q<20 \%$ in the optical band. Grating-based systems offer up to 32 parallel energy channels, ${ }^{8}$ each readout by an independent PMT, but the instrument sensitivity remains limited by the photocathode's thermionic dark noise count rate $-B \geqslant 10$ counts $\mathrm{cm}^{-2} \mathrm{~s}^{-1}$ at room temperature. ${ }^{9}$ More commonly, the fluorochromes are addressed sequentially, repeatedly scanning the sample and registering the fluorescence through a series of narrow band output filters. Multiple scanning increases the possibility of photobleaching.

The low throughput of dispersive spectrometers was one of the motivations for the development in astronomy in the 1990's of cryogenic, energy-resolving detectors such as the STJ and the transition edge sensor (TES). ${ }^{10}$ A STJ consists of two superconducting layers held at a temperature $T$ well below their superconducting-to-normal transition temperature $T_{c}$, and separated by an insulating aluminum oxide layer [see upper inset to Fig. 1(a)]. For $T \sim T_{c} / 10$, the thermally induced tunnel current is negligible and the absorption of a photon of wavelength $\lambda(\mathrm{nm})$ gives rise to a number $N_{0} \sim 7$ $\times 10^{5} / \lambda \Delta(T)$ of excess free charges (quasiparticles) where $\Delta$ $(\mathrm{meV})$ is the superconducting energy gap. ${ }^{4}$ The intrinsic (or "tunnel limited") resolving power $R=\lambda / \Delta \lambda$ of a symmetrical STJ is then

$$
R=357(\lambda \Delta)^{-1 / 2}[1+F+1 /\langle n\rangle]^{-1 / 2},
$$

where $F$ is the Fano factor $(\sim 0.22$ for transition metal 

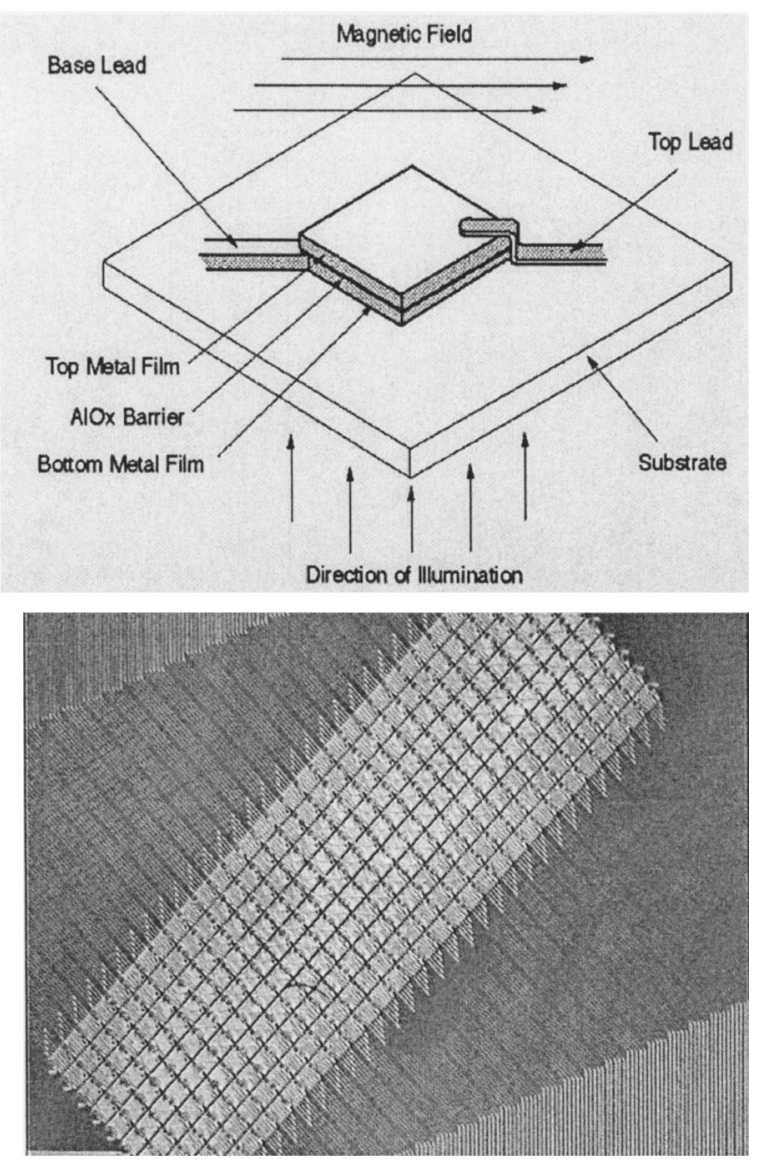

FIG. 1. (a) Schematic representation of single pixel STJ. Optical photons are incident through a transparent substrate on to the lower electrode of a superconductor-insulator-superconductor structure, breaking Cooper pairs and causing a current to flow across the thin $(\sim 10 \AA)$ insulating barrier between the metal films. A magnetic field in the plane of the detector suppresses the Josephson supercurrent of bound Cooper pairs. (b) Nomarski microscope image of $10 \times 32$ pixel molybdenum STJ array. Each pixel is 30 $\mu \mathrm{m}$ on a side.

superconductors ${ }^{6}$ ) and $\langle n\rangle$ is the average number of times that each quasiparticle tunnels across the barrier.

\section{MEASUREMENTS}

The detector used in our initial study of biological fluorescence was a single $30 \times 30 \mu \mathrm{m}^{2}$ STJ with $100 \mathrm{~nm}$ thick Ta layers and $30 \mathrm{~nm}$ thick $\mathrm{Al}$ layers on either side of the tunnel barrier. The transition temperature for bulk tantalum is $4.5 \mathrm{~K}$ and the energy gap $\Delta(0)$ is $0.69 \mathrm{meV}$. The detector was made using photolithographic techniques from a $\mathrm{Ta} / \mathrm{Al}$ multilayer deposited on a polished sapphire substrate. The low leakage current $\left(<0.1 \mathrm{pA} / \mu \mathrm{m}^{2}\right)$ gave a negligible contribution to the baseline noise floor, while the high transmission of the barrier resulted in high signal amplitudes. The detector had a responsivity $\langle n\rangle N_{0} \sim 40000$ tunneled electrons per $\mathrm{eV}$ of photon energy, and a pulse decay time of $\sim 20 \mu \mathrm{s}$. Equation (1) then indicates resolving powers of 16.0 and 8.8 at wavelengths of 600 and $2000 \mathrm{~nm}$. Calibration using monochromatic radiation indicated $R(600 \mathrm{~nm}) \sim 13.3$. Cooling to 300 $\mathrm{mK}$ in a ${ }^{3} \mathrm{He}$ cryostat (i.e., $T \sim T_{c} / 15$ ) kept the thermally excited quasiparticle current well below the leakage current level. We would then expect the dominant background source in the STJ to be cosmic ray muons interacting with the sapphire substrate, in a complex manner, to create pairbreaking phonons at an upper limit rate $B \sim 0.01 \mathrm{~cm}^{-2} \mathrm{~s}^{-1}$. Given a peak STJ quantum efficiency $Q(600 \mathrm{~nm})$ of $75 \%,{ }^{4}$ we then arrive at the sensitivity advantage of the cryogenic detector over a PMT-based system:

$$
\frac{\left(Q_{\mathrm{STJ}} / \sqrt{B}_{\mathrm{STJ}}\right)}{\left(Q_{\mathrm{PMT}} / \sqrt{B}_{\mathrm{PMT}}\right)}=120 \text { times. }
$$

This figure could ultimately be higher in a many-pixel STJ array, where substrate events would give rise to simultaneous pulses in adjacent pixels.

Light from a Leica Aristoplan epifluorescence microscope with a $100 \mathrm{~W}$ Ushio HBO mercury excitation source was coupled into the backilluminated STJ via a $6 \mathrm{~m}$ Oriel 77530 UV-grade fused silica fiber, bandpass $\lambda \sim 200-2000$ $\mathrm{nm}$. The STJ quantum efficiency exceeds $50 \%$ for $\lambda$ values between 150 and $700 \mathrm{~nm}$. No efforts were made to focus the light from the specimen onto the fiber or from the end of the fiber onto the detector. The coupling efficiency was therefore extremely low $(<0.01 \%)$. The detector was held within a light tight shield at the base system temperature of $300 \mathrm{mK}$. The warm end of the optical fiber, however, constitutes a 300 $\mathrm{K}$ black body source which, when convolved with the fiber transmission, provides an effective reference signal in all STJ spectra at a wavelength of $2 \mu \mathrm{m}$ (see Figs. 2 and 4).

The warm readout electronics consisted of a charge sensitive preamplifier situated $\sim 1 \mathrm{~m}$ from the detector, a shaping amplifier (10 $\mu$ s shaping time) and an analog to digital (AD) converter whose output was optically coupled to a PC, on which the pulse amplitude and decay time was recorded for each detected photon. A pulse risetime window was applied to select valid events.

Fluorescence spectra were measured from eleven different, commercially available, labeled uridine nucleotides. Six different substrates were also examined. Four excitation filters were used: the spectra presented here were obtained with the following two filter sets:

(i) Leica filter set I3; 450-490 nm excitation, $510 \mathrm{~nm}$ dichroic cutoff, and $515 \mathrm{~nm}$ long-pass filter.

(ii) Omega Optical Inc. ${ }^{11} 56$ triple excitation filter, with transmission bands centered on 457, 528, and $633 \mathrm{~nm}$, its associated dichroic mirror and an Omega $580 \mathrm{~nm}$ long-pass emission filter.

The transmissions of the actual filters used were confirmed post facto using a laboratory spectrophotometer.

\section{ANALYSIS}

Even though excited far from maximum absorbance, spectra were obtained from fluorochromes emitting in the red (Alexa 594, Fluorored, and Texas Red) and green (Fluorogreen, Avidin FITC, and Alexa 488). We illustrate the potential of the STJ for fluorescent imaging with particular reference to the dyes Alexa 488 and Alexa 594. ${ }^{12}$ Figure 2 shows the pulse height spectrum of the latter fluorochrome with the Leica I3 filter set. The spectrum is cutoff at $2.4 \mathrm{eV}(515 \mathrm{~nm})$ by the long-pass filter. Even with the fluorochrome excited at only $\sim 3 \%$ of maximum absorbance, the signal-to- 


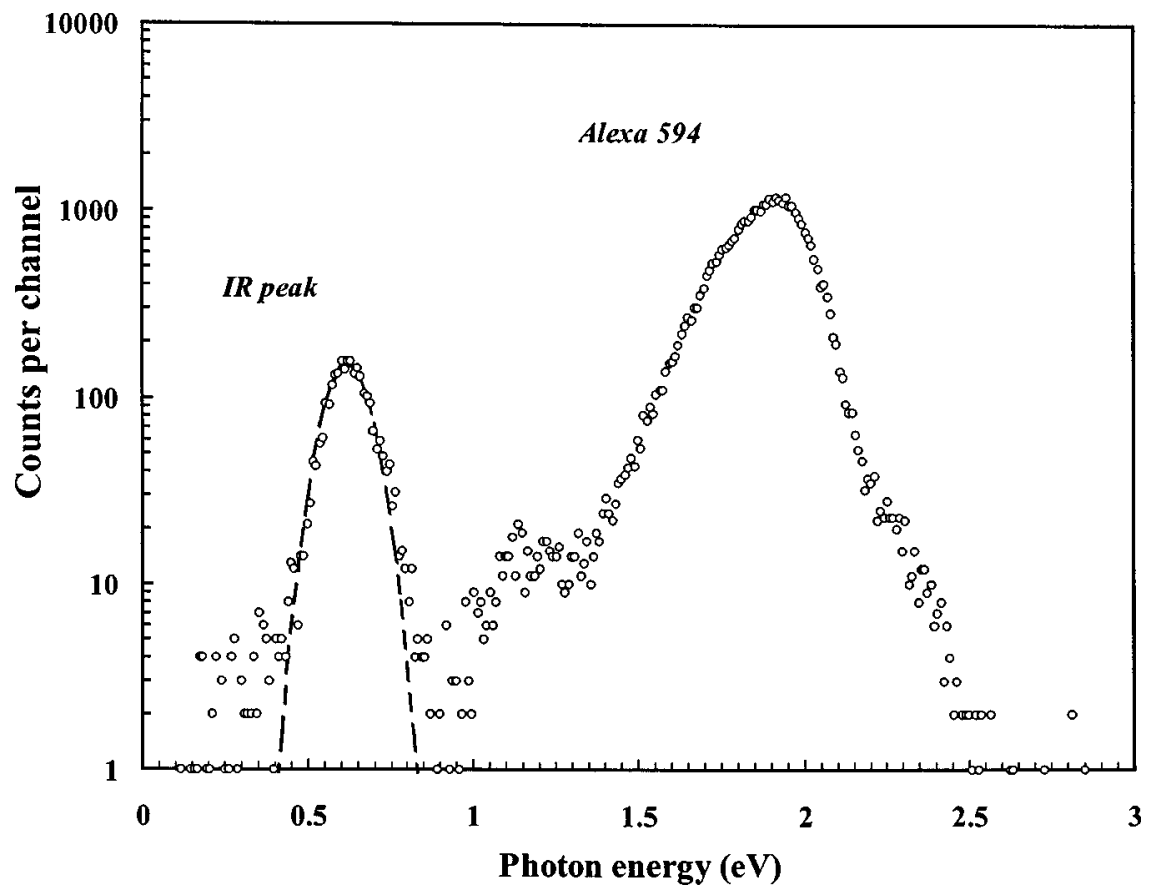

FIG. 2. Tantalum STJ pulse height spectrum on a logarithmic scale for the fluorochrome Alexa 594, obtained using the Leica I 3 filter set. The broken curve is a Gaussian fit to the peak at $0.62 \mathrm{eV}$ arising from the infrared emission from the warm optical fiber which channels light from the microscope stage into the cryostat.

background (peak channel count divided by average channel count at energies just above the IR peak) is at least 275:1. Fitting a normal distribution (the broken curve) to the IR peak indicates $R(2000 \mathrm{~nm})=4.0$. Injecting a known charge to the warm STJ preamplifier from an electronic pulser produced a peak width of $0.084 \mathrm{eV}$ FWHM. The quadrature sum of tunnel limit and electronic noise contributions then implies a $2 \mu \mathrm{m}$ resolving power $R=5.7$, slightly better than observed.

For each fluorochrome, the microscope focus was adjusted to produce an output count rate of $800 \mathrm{~Hz}$ - the maximum rate before pulse pileup compromised the spectral resolution. The "dark" count rate obtained by closing the microscope shutter between bulb and filter was $0.09 \mathrm{~s}^{-1}$. Because individual spectra were not obtained at constant intensity, overlaying two STJ spectra does not necessarily represent the degree of spectral separation for the corresponding mixture of fluorochromes. Nevertheless, Fig. 3 indicates that, while the degree of spectral overlap is significant, it is straightforward to deconvolve the relative intensity contributions of mixed Alexa 488 and Alexa 594 probes. We note that the peak emission energies and spectral widths for both probes differ significantly from the templates published by the manufacturer. ${ }^{12}$ The peak emission energy for Alexa 594 is redshifted by $0.17 \mathrm{eV}$ from the expected value of 2.088 $\mathrm{eV}$; that of Alexa 488 , by $0.29 \mathrm{eV}$. The measured width of the Alexa 594 distribution is somewhat larger than obtained from the convolution of the manufacturer's spectrum with the energy response of the STJ. Since the STJ calibration rules out any "zero shift" in the detector response (see Fig. 4), we conclude that these measurements constitute evidence for the influence of local environment on fluorochrome emis-

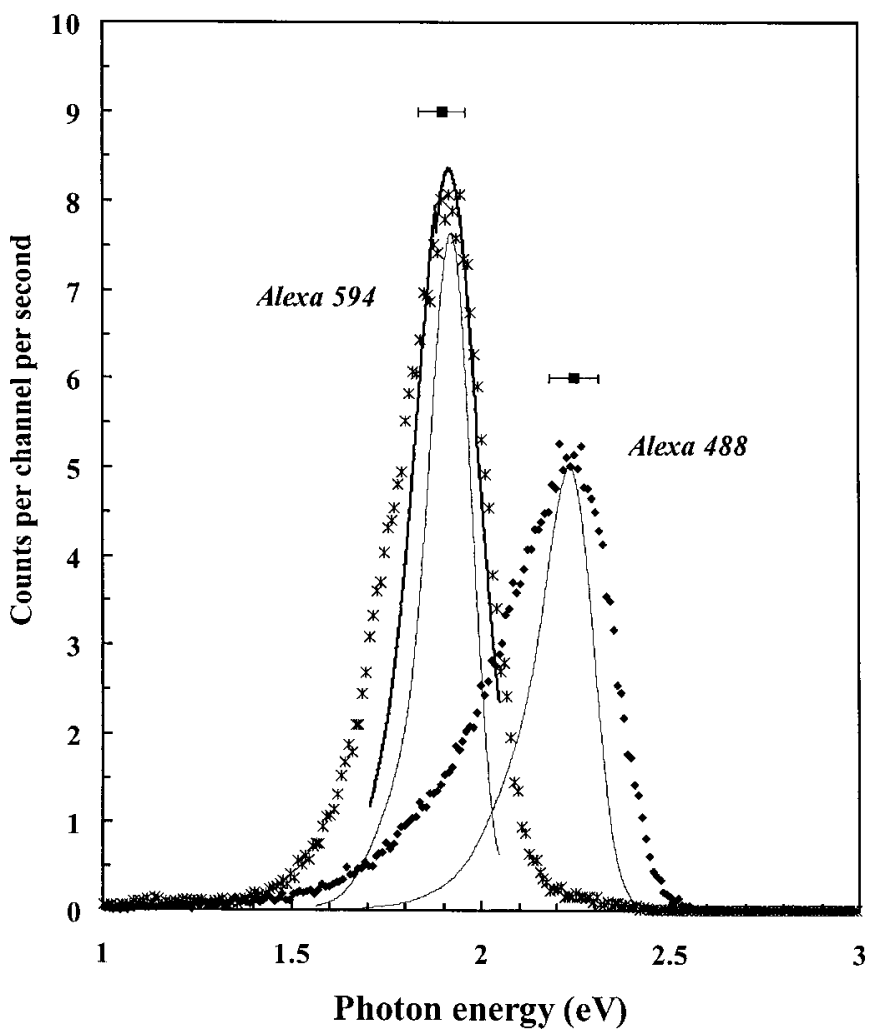

FIG. 3. Overlaid tantalum STJ pulse height spectra for the fluorochromes Alexa 594 (stars) and Alexa 488 spectra (diamonds). Spectra were obtained using the Leica I 3 filter set. The full feint curves are the manufacturer's published emission spectra, corrected for optical fiber transmission and STJ quantum efficiency. The finite resolving power of the detector is indicated by FWHM energy resolution "error bars." The published emission spectra have been shifted in energy so that their peaks coincide with our measured peaks. The solid curve is the convolution of the manufacturer's Alexa 594 spectrum with the Gaussian energy response function of the STJ. 


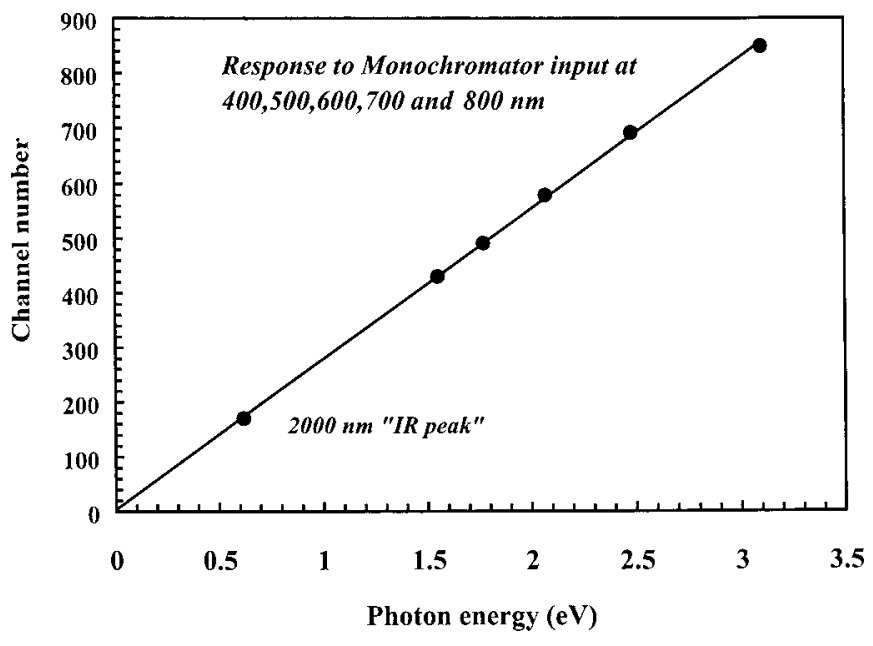

FIG. 4. STJ photon energy-to-pulse height calibration graph, obtained from monchromatic input at five separate wavelengths. Using the peak due to long-wavelength emission from the silica fiber, we see that excellent detector linearity is preserved into the infrared.

sion. Here, the dyes dried out from solution spotted onto glass cleaned with chromic acid.

Figure 5 shows the Alexa 594 and Alexa 488 spectra obtained with the Omega triple filter set; the "top hat" transmission bands of the output filter are indicated by the broken vertical lines. This figure shows that the spectroscopic capability of the STJ may be combined with appropriate selections of input and output filters to give highly preferential registration of one fluorochrome. In band 1, centred on a photon energy of $2.5 \mathrm{eV}$, the ratio of peak intensities is about $10: 1$, while in band 2 at $2.8 \mathrm{eV}$, the ratio is $1: 1$.

Figure 6 illustrates the first application of the STJ to the study of genetic material hybridized, on an Arrayit ${ }^{\mathrm{TM}}$ (Ref. 13) substrate, with multiple probes (Alexa 568, Alexa 594, and Bodipy 630/650 - all produced by Molecular Probes Inc. ${ }^{12}$ ). Data set 1 represents the measured sample spectrum, while curves 2, 4, 5, and 6 are the component spectra measured for the three fluorochromes separately and for the substrate. Curve 3 indicates that a linear sum of the component intensities with weights:

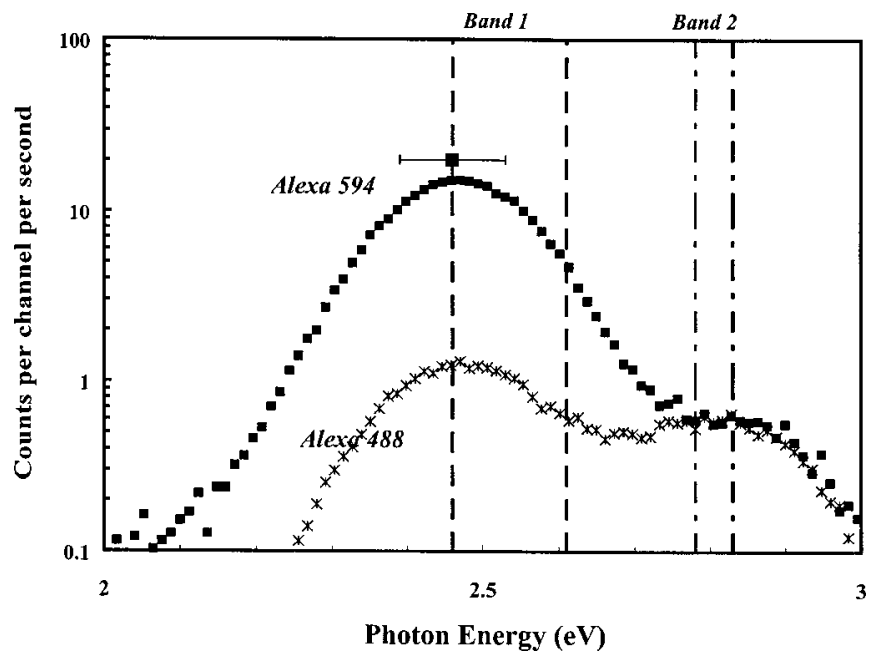

FIG. 5. Overlaid Alexa 594 and Alexa 488 pulse height spectra measured with the adapted Omega filters. The broken vertical lines indicate the two filter pass bands; the bar indicates the FWHM energy resolution of the detector at the peak of the Alexa 594 response.

\section{Alexa 568:Bodipy 630/650:Substrate:Alexa 594}

$$
=10: 10: 4: 3 \text {, }
$$

provides an excellent fit to the data in the $1.9-2.4 \mathrm{eV}(515-$ $652 \mathrm{~nm}$ ) band. We cannot attribute the excess intensity at lower energies to the presence in the sample of unhybridized DNA, since the intrinsic fluorescence of DNA is well known to peak in the UV. One explanation is excimer (excited dimer) emission arising as a result of the high concentrations of the fluorochrome probes in the sample, although fluorescence from components of the solutions used to prepare the array may also be contributing. The manufacturer's handbook for Bodipy dyes states that these fatty acid analogs do generally exhibit concentration-dependent excimer formation and consequent redshifting of their fluorescence. ${ }^{12}$

\section{DISCUSSION}

We have shown that a cryogenic detector can detect, quantitatively and at very high signal-to-noise compared to

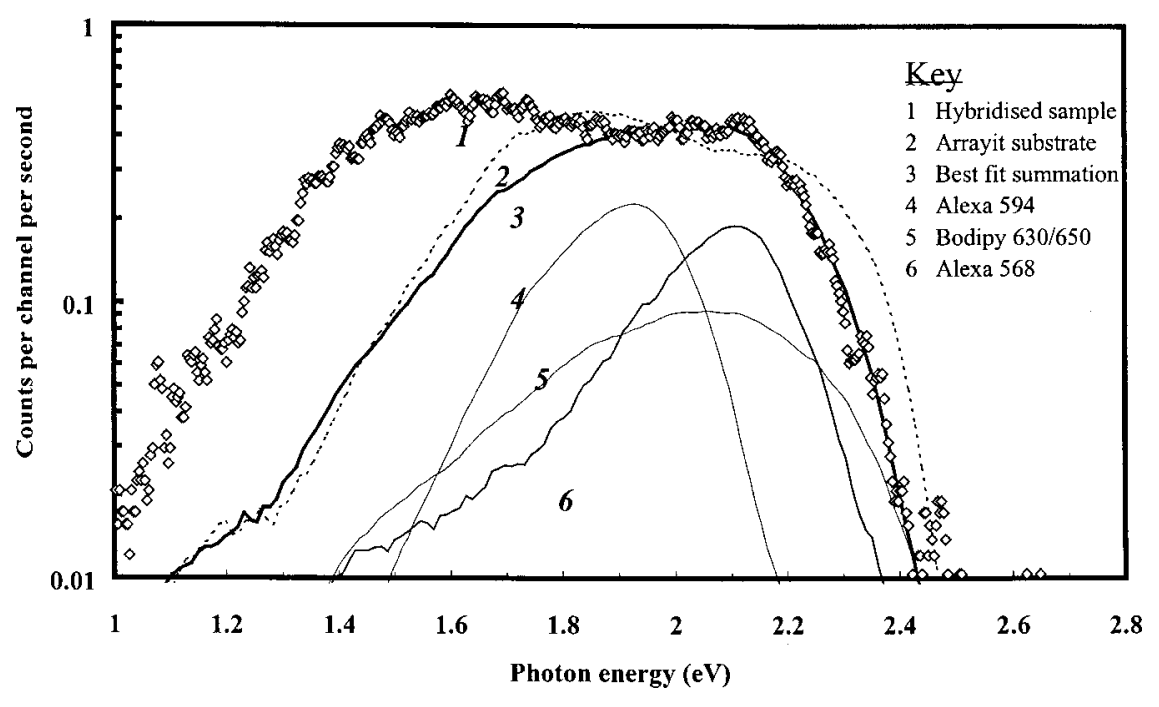

FIG. 6. Composite fluorescence spectrum from genetic material hybridized to three separate probes-Alexa 568 , Alexa 594, and Bodipy 630/650. The fitting of a weighted linear sum of the individually measured responses of the three fluorochromes and the substrate is described. 
conventional systems, characteristic fluorescent emission spectra from fluorochromes commonly used in biology. Even with single excitation wavelength bands, photon-by-photon registration of the complete spectrum allows separation of multiple fluorochromes. Remarkably, we found that nonfocused, low levels of peripheral sample illumination with Philips TLD 36W/83 fluorescent tubes (i.e., normal laboratory lighting) were able to excite count rates in excess of $1000 \mathrm{~Hz}$ from fluorochromes on a slide on a microscope stage.

The present Ta technology $(R \sim 10-20)$ is compatible with at least four simultaneous labels with a single excitation filter; the potential of $\mathrm{Hf}[\Delta(0)=0.02 \mathrm{meV} ; R \sim 80]$ and Mo $[\Delta(0)=0.14 \mathrm{meV} ; R \sim 40]$ for better resolution (at lower operating temperatures) is well documented. ${ }^{4}$ In the context of biological assays, in particular the measurement of hybridization of labeled probes to microarrays, any increase in the number of labels that can be measured without interference is important because either increased numbers of internal controls can be included, or more samples can be tested simultaneously. The modest count rate limitation of a single STJ pixel can be overcome by using parallel arrays; $6 \times 6$ element Ta STJ matrices have been fabricated and the development of $10 \times 32$ Mo arrays studied by the ESTEC Group [see Fig. 1(b)]. These developments and the production of closed cycle cooling systems for the $T<100 \mathrm{mK}$ regime will have an important bearing on the ultimate utility of optical STJs in the life sciences.

\section{ACKNOWLEDGMENT}

The authors acknowledge the support of Muna Naji in reducing the STJ datasets.

${ }^{1}$ T. Schwarzacher and J. S. Heslop-Harrison, Practical In Situ Hybridization (Bios, Oxford, 2000), p. 213.

${ }^{2}$ M. A. C. Perryman, C. L. Foden, and A. Peacock, Nucl. Instrum. Methods Phys. Res. A 325, 319 (1993).

${ }^{3}$ A. Peacock, P. Verhoeve, N. Rando, A. van Dordrecht, B. G. Taylor, C. Erd, M. A. C. Perryman, R. Venn, J. Howlett, D. J. Goldie, J. Lumley, and M. Wallis, Nature (London) 381, 135 (1996).

${ }^{4}$ P. Verhoeve, N. Rando, A. Peacock, A. van Dordrecht, A. Poelaert, and D. Goldie, IEEE Trans. Appl. Supercond. 7, 3359 (1997).

${ }^{5}$ N. Rando et al., Rev. Sci. Instrum. 71, 4582 (2000).

${ }^{6}$ N. Rando, A. Peacock, A. van Dordrecht, C. L. Foden, R. Engelhardt, B. G. Taylor, J. Lumley, and C. Pereira, Nucl. Instrum. Methods Phys. Res. A 313, 173 (1992).

${ }^{7}$ A. H. Sommer, Photoemissive Materials (Wiley, New York, 1968).

${ }^{8}$ META detector for the Carl Zeiss LSM 510 Confocal Microscope. Carl Zeiss, Welwyn Garden City, Herts, UK.

${ }^{9}$ I. G. Butler et al., Proc. SPIE 2278, 126 (1994).

${ }^{10}$ R. W. Romani, A. J. Miller, B. Cabrera, S. W. Nam, and J. M. Martinis, Ap. J. (in press).

${ }^{11}$ Omega Optical Inc., 210 Main St., Brattleboro, VT 05301.

${ }^{12}$ N. Panchuk-Voloshina et al., J. Histochem. Cytochem. 47, 1179 (1999); Molecular Probes Inc., P.O. Box 22010, Eugene, OR 97402-0469.

${ }^{13}$ TeleChem International Inc., 524 E. Weddell Dr., Suite No. 3, Sunnyvale, CA 94089 . 